\title{
206. Klinische Erfahrungen mit der Nierentransplantation
}

\author{
F. W. EigleR *, R. Nagel, V. Zühlke, D. SachWeh, H. Lauschke, \\ J. Eigler, K. Fincke, E. Renner, H. G. Sieberth und \\ W. HÜBNER-Köln
}

\section{Clinical Experience in Renal Transplantation}

Summary. Between June 1967 and March 1971, 21 allogenic renal transplantations, in 19 patients, were carried out at the Surgical University Clinic, Cologne. 14 of these patients are still alive, of whom 12 have "sufficient" or "good" renal function and 2 are back in dialysis programm. Fatal complications were pneumonia in three cases (one caused by the cytomegaly virus), cerebral oedema after hyper. acute rejection with malignant hypertension in one case, and in one case probably an acute oxalosis following a second transplantation, with renewed hyper-acute rejection. The most common local surgical complication was a urinary fistula which occurred after four of the transplantations. Of the general complications a triple jejunal perforation, probably due to cortisone treatment, is of particular interest. Based on our own experience and the relevant literature, there would seem to be two important rules in kidney transplantations:

1. No transplantation without a negative cross-test.

2. A fresh or a well-preserved kidney is better than one that is merely suitable according to leucocytegroup tests.

With these principles of selection we hope to maintain or even improve on our satisfactory results of 1970 (only one death and seven functioning kidneys out of ten renal transplantations).

Zusammenfassung. Vom Juni 1967 bis März 1971 wurden an der Chirurgischen Universitätsklinik Köln-Lindenthal 21 allogene Nierentransplantationen bei 19 Patienten durchgeführt. 14 Patienten überleben, 12 davon mit ausreichender bis guter Nierenfunktion, 2 sind zurück im Danerdialyseprogramm. Als tödliche Komplikationen traten $3 \mathrm{mal}$ eine Pneumonie auf (davon einmal mit CytomegalieVirus), einmal ein Hirnödem nach hyperakuter Abstoßung mit maligner Hypertonie und einmal wahrscheinlich eine akute Oxalose nach einer zweiten Transplantation mit erneuter hyperakuter Abstoßung. An lokalen chirurgischen Komplikationen war die Harnfistel nach 4 Transplantationen am häufigsten. An allgemeinen Komplikationen scheint eine dreifache Jejunumperforation, wahrscheinlich als Folge der Cortison-Medikation, besonders bemerkenswert. Auf Grund der eigenen Frfahrungen und entsprechender Literaturhinweise scheinen uns z. Z. 2 Gesichtspunkte für die Nierentransplantation besonders wichtig: 1 . keine Transplantation ohne negativen Kreuztest, 2. eine lebensfrische, evtl, gut konservierte Niere ist besser als eine lediglich gut nach Leukocytengruppen getestete. Mit diesen Grundsätzen hoffen wir, die guten eigenen Ergebnisse des Jahres 1970 (nur 1 Todesfall und 7 funktionierende Nieren bei 10 Transplantationen) beibehalten bzw. noch verbessern zu können. 